(C) Wuhan Institute of Virology, CAS and Springer-Verlag Berlin Heidelberg 2011

\title{
Indinavir Resistance Evolution: a Comment
}

\author{
Viroj Wiwanitkit ${ }^{* *}$ \\ (Wiwanitkit House, Bangkhae, Bangkok 10160,Thailand)
}

\section{Dear Editor,}

I read the recent report on HIV with a great interest ${ }^{[2]}$. Geng et al concluded that "Indinavir- resistance evolution was observed by single-genome amplification ${ }^{[2] " ~ a n d ~ " D u r i n g ~ t h e ~ c o u r s e ~ o f ~ c h a n g i n g ~ t h e ~ r e g i m e n ~ t o ~}$ incorporate Indinavir, the G73S mutation occurred and was combined with M46I/ L90M ${ }^{[2]}$." I have some points for discussion on this work. First, focusing on the single-genome amplification, although it is acceptable the false positive can still be detected ${ }^{[3]}$. It is interesting to compare the result from this Stanford HIV Drug Resistance Database-based study to modified phenotypic drug susceptibility assay-based study, which was reported to provide discordant result ${ }^{[1]}$. Second, it is interesting which biological process lead to the change of the resistant pattern on the passed time. The situation of cross resistance should also be investigated since it was reported to be common $^{[4]}$.

\section{References}

1. Choi J Y, Kwon O K, Choi S Y, et al. 2010. Drug susceptibility of human immunodeficiency virus type 1-derived pseudoviruses from treatment-experienced patients to protease inhibitors and reverse transcriptase inhibitors, using a modified single-round assay. J Clin Virol, [Oct 20. Epub ahead of print]

2. Geng Q M, Li H P, Bao Z Y, et al. 2010. Indinavir resistance evolution in one human immunodeficiency virus type 1 infected patient revealed by single-genome amplification. Virol Sin, 25 (5): 316-328.

3. Pushkarev D, Neff N F, Quake S R. 2009. Single-molecule sequencing of an individual human genome. Nat Biotechnol, 27 (9): 847-852.

4. Rhee S Y, Taylor J, Fessel W J, et al. 2010. HIV-1 protease mutations and protease inhibitor cross-resistance. Animicrob Agents Chemother, 54 (10): 4253-4261.

Received: 2010-10-28, accepted: 2010-11-16

** corresponding author.

Phone: +66-24132436, Email: wviroj@yahoo.com 